\title{
Indicadores de adicción a las redes sociales y factores de personalidad eficaz en escolares de secundaria de Lima
}

\author{
Indicators of social media addiction and efficient personality factors in high school students in Lima
}

Roberto Bueno Cuadra ${ }^{1}$, Armando Martínez Portillo ${ }^{1}$, Evelyn Barboza Navarro ${ }^{1}$

\section{RESUMEN}

El objetivo fue determinar si existe relación y en qué magnitud entre los factores de personalidad eficaz y los de adicción a las redes sociales en escolares de cuarto y quinto de secundaria. Participaron 304 escolares de dos centros educativos estatales de Lima Metropolitana de 14 a 18 años $(M=15,87 ; D E=0,965)$. Los datos fueron obtenidos mediante el Cuestionario de Personalidad Eficaz (CPE) de Del Buey et al. y el Cuestionario de Adicción a las Redes Sociales (CARS) de Escurra y Salas. El análisis factorial confirmó la estructura de cuatro factores propuesta por Dapelo et al., para la adaptación chilena del CPE, mientras que en el caso del CARS se optó por una estructura de cinco factores. Se encontró diferencias significativas, aunque con tamaño de efecto nulo, entre hombres y mujeres sólo en cuatro de las cinco dimensiones del CARS. Finalmente se hallaron correlaciones negativas (más de la mitad de ellas a nivel de $p<.001$ ) entre los factores de necesidad de conexión, preponderancia y tiempo excesivo, con los cuatro factores de personalidad eficaz.

PALABRAS CLAVE: Adicción a las redes sociales, análisis factorial, confiabilidad, escolares, personalidad eficaz.

\section{SUMMARY}

The objective in this study was to determine whether it exists a relationship and in what amount between factors of efficient personality and factors of social networking addiction in fourth and fifth high school graders in Lima. Participants were students from two schools from Lima Metropolitana, aged 14 to $18(M=15.87 ; S D=0.965)$. Data were obtained through the CPE of Del Buey et al., and the CARS of Escurra and Salas. Factor analysis confirmed a four factor structure, as proposed by Dapelo et al. for the Chilean adaptation of the CPE, meanwhile in the case of the CARS we chose a five factor structure. Significant differences were found, though with null effect size, between males and females on four of the five dimensions of the CARS. Finally, we found significant negative correlations (more than the half of the in the level of $p<.001$ ) between factors of necessity of connection, salience and excessive time of connection, and the four factors of efficient personality.

KEY WORDS: Social networking addiction, factor analysis, reliability, high school students, efficient personality.

\section{INTRODUCCIÓN}

El desarrollo tecnológico conlleva un aporte al bienestar de los seres humanos en la medida que el mismo provee de más y mejores herramientas para el logro de múltiples objetivos: desde realizar las actividades cotidianas hasta resolver importantes problemas prácticos en el sector productivo, la educación, la salud y muchas áreas más. No obstante, también es frecuente que la difusión de una nueva tecnología acarree problemas sociales inesperados. Uno de estos problemas consiste en el abuso de tales medios tecnológicos, de tal forma que, en algunos casos, podría configurarse un conjunto de

\footnotetext{
Facultad de Psicología, Universidad Nacional Federico Villarreal. Lima, Perú
} 
comportamientos propios de una adicción. Esto es lo que parece ocurrir con internet en general, y con las redes sociales digitales en particular. Las redes sociales digitales, o redes sociales, a secas, pueden definirse como "comunidades virtuales donde los usuarios pueden crear perfiles públicos, individuales, interactuar con amigos de la vida real y conocer otras personas que comparten los mismos intereses (Griffiths, Kuss y Demetrovics, 2014, p. 119). Son espacios donde uno puede darse a conocer y compartir gustos, información y experiencias y todo ello de manera compartida, ya que los demás usuarios pueden también comentar lo presentado en dicho perfil (Boyd y Ellison, 2007; Carbonell, 2015).

Las redes sociales sirven principalmente a propósitos de socialización, y por otro lado, encuentra en los adolescentes y jóvenes entre sus más frecuentes y entusiastas usuarios. Si bien se pondera en alta estima la importancia y utilidad en la vida contemporánea de las redes sociales como elemento de comunicación, socialización y entretenimiento (Barros et al., 2017; Caldevilla, 2010; Sanz, Alonso, Sáenz, Ponce de León, Valdemoros, 2018), la literatura ya menciona también que, en ciertos casos, la participación en ellas asume la forma de un comportamiento adictivo (Chóliz y Villanueva, 2011; Echeburúa y de Corral, 2010; Fernández, 2013; García, 2013; Kuss y Griffiths, 2017). Como ya se ha señalado repetidas veces, las redes sociales tienen todas las características para convertirse en un elemento altamente atractivo y recompensante para los usuarios más jóvenes, lo que incluye su potencial para satisfacer necesidades de gran importancia en ese grupo de edad (Echeburúa y Requesens, 2012; Ross et al., 2006).

Siguiendo los criterios de West y Brown (2013) sobre la definición de adicción, se puede hablar de adicción a las redes sociales siempre que el individuo pierda el control sobre sus hábitos de conexión y que dichas acciones sean reiterativas a pesar de que ello pueda acarrear consecuencias adversas. Son comunes los reportes acerca de las varias consecuencias negativas que se siguen de este descontrol sobre el uso de las redes sociales (e. g., Andreassen, 2015; Griffiths et al., 2014); por ejemplo, Cam e Isbulam (2012), investigando la adicción a Facebook en estudiantes turcos, observaron entre aquellos que tenían una alta puntuación en este factor, características como una disminución en diversas actividades sociales, problemas académicos y laborales, menos intimidad con sus parejas y quejas de otros sobre su uso de Facebook, entre otros aspectos. Si bien es necesario hacer una distinción entre "uso excesivo", y una adicción propiamente dicha, no puede dejar de mencionarse que ciertas evidencias indican que el uso excesivo es predictor de adicción a las redes sociales (Wan, 2009). Los estudios a nivel internacional, citados por Andreassen (2015) indican tasas de prevalencia mayores a $1 \%$ y en algunos casos, llegando a $8.6 \%$, como sucede con estudiantes de pre-grado usuarios de Facebook en el Perú, según un estudio publicado el 2013, citado por Andreassen.

Se han señalado algunos de los factores de riesgo para el desarrollo de una adicción a las redes sociales. Echeburúa (2012) enumeró entre ellos a factores de "vulnerabilidad psicológica". Esto implica la presencia de ciertos factores individuales que incrementan el riesgo de aparición de algún desorden emocional o conductual, como en este caso, una posible adicción a las redes sociales. A esta vulnerabilidad pueden quedar asociados ciertos factores sociales, por ejemplo, Grifftihs (1996) menciona que una adicción puede mantenerse debido a los lazos sociales con otras personas que tienen esa misma adicción.

Entre los factores de vulnerabilidad que podrían estar fuertemente vinculados a la presencia de indicadores de adicción a las redes sociales, debemos considerar seriamente a los aspectos de personalidad del individuo, entendiendo por personalidad a los estilos de comportamiento que son propios y diferentes de un individuo a otro y que son consistentes en el tiempo. Podemos partir del hecho de que ciertos factores de personalidad están efectivamente relacionados con determinadas actividades realizadas en redes sociales como Facebook (e. g., Eftekhar, Fullwood y Morris, 2014; Yesil, 2014). Asimismo, Wilson, Fornasier y White (2010), investigando en universitarios australianos, hallaron que una alta extraversión y baja responsabilidad predice el tiempo usado en las redes sociales. Sin embargo, como sucede con otras adicciones, las tendencias individuales de conducta asociadas en mayor o menor medida con el desarrollo de la adicción tienen que ver, en gran medida, con una mayor vulnerabilidad al estrés; es decir, con una mayor probabilidad de que los eventos estresantes provoquen alguna clase de disfunciones emocionales o conductuales. En unión de otros factores, estas condiciones pueden, a su vez, desencadenar problemas adictivos, entre ellos el uso excesivo y descontrolado de las redes sociales. De hecho, varios estudios confirman que un uso más prolongado e intenso de las redes sociales está asociado a un mayor distrés (estrés excesivo) psicológico (e. g., Müller et al., 2016), aun 
cuando la relación entre el uso prolongado de las redes sociales e indicadores de salud mental es todavía materia de controversia (Pantic, 2014).

Varios estudios confirman, a nivel global, que, efectivamente, ciertos aspectos de la personalidad sí están vinculados con el uso problemático o con indicadores de adicción a las redes sociales. Por ejemplo, el neuroticismo y la extraversión (McCrae y Costa, 2006), parecen estar muy relacionados con el desarrollo de la adicción a las redes sociales. El neuroticismo se define como el grado en que el estrés puede conducir al individuo a estados de ansiedad y depresión, en tanto que la extraversión se refiere principalmente a aspectos de impulsividad, energía y sociabilidad. Andreaessen, Tosheim, BrunBerg y Pallesen (2012) encontraron que los indicadores de adicción a las redes sociales correlacionaron positivamente con rasgos de neuroticismo $y$ extraversión y negativamente con responsabilidad. También el factor de responsabilidad está asociado con la adicción a Facebook (Blachnio, Przepiorka, SenolDurak y Sherstyuk, 2017). Sin embargo, algunos otros estudios no han confirmado la relación entre grandes dimensiones de la personalidad, como la extraversión y el neuroticismo, e indicadores de adicción a internet (Franco, Martínez, Parra y Ramos, 2018). Estas dimensiones engloban un amplio conjunto de rasgos por lo que su dinámica y relaciones pueden ser de distinta naturaleza que en el caso de rasgos más específicos.

Nuestro planteamiento es que algunos rasgos de personalidad pueden constituir factores protectores, conduciendo a una menor vulnerabilidad psicológica, en tanto que otros actúan más bien como factores de riesgo. Uno de ellos es la autoestima, la cual puede definirse como "la evaluación positiva o negativa de un individuo acerca de sí mismo" (Smith, Mackie y Claypool, 2014, p. 107). La baja autoestima está asociada con el "uso patológico" de internet (Niemz, Griffiths y Banyard, 2005) y con la tendencia adictiva hacia la actividad de mensajería instantánea (Ehrenberg, Juckes, White y Walsh, 2008). La baja autoeficacia y el locus de control externo están, a su vez, relacionados con la adicción a internet (Iskender y Akin, 2010). Ceyhan y Ceyhan (2008) investigaron a 559 universitarios turcos y encontraron que entre la soledad, la depresión y la autoeficacia relacionada con el uso de computadoras, la primera era la principal predictora de "uso problemático" de internet. En otro estudio, realizado en 371 estudiantes británicos, también se comprobó que una más baja autoestima se asociaba a mayores niveles de "uso patológico" de internet (Niemz, Griffiths y Banyard, 2005). Las personas con baja autoestima tienden a usar las redes sociales como un medio para mejorar su autoestima (e. g. (Błachnio, Przepiorka y Rudnicka, 2016; Gonzales y Hancock, 2011; Steinfield, Ellison, \& Lampe,2008). Asimismo, una autoestima más baja está relacionada con mayor actividad en red (Mehdizadeh, 2010).

Precisamente, el constructo de personalidad eficaz intenta capturar esas características "positivas" vinculadas al afrontamiento, autoestima, autoeficacia $\mathrm{y}$, en general, rasgos que identifican la conducta proactiva y asertiva y orientada hacia la solución de problemas (Martín del Buey et al., 2015; Martín del Buey, Martín \& Fernández, 2009). Rasgos de la personalidad eficaz son los siguientes: "Autoconcepto, Autoestima, Motivación, Expectativas adecuadas, Atribución de causalidad, Afrontamiento de problemas, Toma de decisiones, Empatía, Asertividad y Comunicación" (Pizarro, Martín \& Cortés, 2012, p. 108). Se considera que las características que definen la personalidad eficaz interactúan entre sí y se encuentran muy correlacionadas unas con otras (Kifafi, Lizana \& Ortiz, 2012). Todas estas son características que actúan promoviendo un mejor bienestar psicológico (Bustamante et al., 2014), y por ello, probablemente, reduciendo el riesgo de disfuncionalidades o desórdenes tanto emocionales como conductuales. Por ejemplo, Blachnio et al., (2016), mostraron, en estudiantes polacos, que los adictos a Facebook tenían autoestima más baja que sus compañeros no adictos. Otros estudios internacionales que indican la asociación entre adicción a las redes sociales y baja autoestima son los de Wilson et al., (2010) y Hong, Huang, Lin y Chiu (2014). Partiendo de este hecho, nuestra hipótesis es que existe una relación inversa entre los factores de personalidad eficaz y determinados indicadores de adicción a las redes sociales. Planteamos, además, que esta relación inversa puede variar en magnitud dependiendo de qué tipo de indicadores de adicciones a las redes sociales se tenga en cuenta. La carencia de información respecto de cómo se relacionan con la adicción a las redes sociales otros aspectos de la personalidad eficaz dificulta el desarrollar una visión global acerca de cómo un conjunto de conductas orientadas a la solución de problemas, el afrontamiento y la proactividad se vinculan con determinados indicadores de adicción a las redes sociales. Es de suponer, a partir de la propuesta teórica, que la personalidad eficaz en su 
conjunto, y en mayor o menor grado, cada una de sus dimensiones, estará negativamente relacionada con tales indicadores.

\section{MATERIAL Y METODOS}

La población estuvo formada por escolares de un colegio estatal mixto de Lima, de cuarto y quinto de secundaria. Para el cálculo del tamaño de muestra se tuvo en cuenta criterios de tamaño de efecto y de potencia de la prueba (Polit Y Hungler, 2000). Se asumió un tamaño de efecto mediano (alrededor de $\mathrm{r}=0,30)$ para estudios de correlación de variables. Considerando un nivel de significación de 0,05 (bilateral) y una potencia de prueba de 0,95 , se estimó el tamaño de muestra en 138 (Machin, Campbell, Tan y Tan, 2018, p. 314); sin embargo, se obtuvo datos de 318 participantes. Se consideró como criterios de inclusión la condición de vivir con ambos padres. De la muestra final se excluyó a los participantes que no llenaron los cuestionarios correctamente y los que manifestaron no tener una cuenta en ninguna red social, quedando finalmente 304 participantes. La edad de dichos escolares variaba de 14 a 18 años $(M=15,87$; $D E=0,965)$. La Tabla 1 presenta la distribución de la muestra según sexo, grado escolar y edad.

\section{Instrumentos}

Cuestionario de Personalidad Eficaz para Adolescentes (CPE), adaptación chilena (Dapelo, Marcone, Martín del Buey, Martín y Fernández, 2006). El instrumento original, de 58 ítems, fue desarrollado por Martín del Buey et al., (2004). La versión que se utiliza en el presente estudio es la adaptación de Dapelo et al., (2006) en adolescentes chilenos. Esta versión consta de 23 ítems, los cuales fueron obtenidos después de eliminarse aquellos ítems que tenían una

Tabla 1. Distribución de la muestra según sexo, grado escolar y edad

\begin{tabular}{llcc}
\hline & & Frecuencia & Porcentaje \\
\hline Sexo & Masculino & 86 & 28,3 \\
& Femenino & 218 & 71,7 \\
Grado & Cuarto & 150 & 49,3 \\
& Quinto & 154 & 50,7 \\
Edad & 14 & 12 & 3,9 \\
& 15 & 110 & 36.2 \\
& 16 & 104 & 34.2 \\
& 17 & 61 & 20.1 \\
& 18 & 17 & 5.6 \\
\hline
\end{tabular}

baja correlación con el total o que habían tenido cargas factoriales menores a .30. El análisis factorial arrojó cuatro factores: 1. Fortalezas del yo (logros, autoconcepto y autoeficacia académica); 2 . Relaciones del yo (asertividad, empatía, habilidad social); 3 . Retos del yo (toma de decisiones, resolución de problemas) y 4. Autoestima. Estos cuatro factores explican el $50,43 \%$ de la varianza total de las puntuaciones. Un análisis factorial de segundo orden produjo un único factor que explica el 43,28\% de la varianza total. Los ítems se responden mediante una escala tipo Likert que va desde "Nunca" (1 punto) a "Siempre" (5 puntos). La confiabilidad (alfa de Cronbach) de la puntuación total es 0,84 y las de las cuatro escalas oscila entre 0,72 y 0,80 . En el presente trabajo se realizó un nuevo estudio de confiabilidad y de validez factorial.

Cuestionario de Adicción a Redes Sociales (CARS) de Escurra y Salas (2014): Fue desarrollado en Lima a partir de los indicadores de adicción a sustancias del DSM-IV. El cuestionario fue sometido primero a evaluación por jueces y la versión resultante se aplicó a una muestra de 18 universitarios. Una vez revisado, el instrumento fue sometido a análisis factorial exploratorio, evaluación de la confiabilidad y análisis factorial confirmatorio. Este análisis arrojó tres dimensiones: 1. Obsesión por las redes sociales; 2. Falta de control personal en el uso de las redes sociales y 3. Uso excesivo de las redes sociales. La confiabilidad de las puntuaciones de las escalas oscila entre 0,88 y0,92. El instrumento está constituido por 24 ítems tipo Likert que se puntúan en una escala que va de nunca a siempre, que se califican de 0 a 4 respectivamente. Un mayor puntaje indica mayor intensidad en el factor de adicción. Se reporta en el este trabajo un nuevo estudio de confiabilidad y validez factorial de este instrumento.

\section{Procedimiento}

Los instrumentos fueron aplicados de forma colectiva, durante la misma sesión y en el salón de clase durante el horario normal de clase. Las respuestas fueron anónimas. A los participantes se les indicó verbalmente las instrucciones y se les recalcó la importancia de que respondan con veracidad. En todos los casos, los padres o tutores firmaron el consentimiento informado y los escolares hicieron lo propio con el documento de asentimiento informado.

\section{Análisis de datos}

Para el procesamiento de datos se utilizó el paquete 
estadístico SPSS 25. En primer lugar se calcularon las correlaciones ítem-test de cada uno de los cuestionarios, considerándose retener los ítems cuya correlación con el test fuera de al menos 0,20. Seguidamente, se realizaron las pruebas de Kaiser-Meyer-Olkin (KMO) y de Bartlett para verificar la factibilidad de realizar el análisis factorial en cada uno de los instrumentos. Para el análisis factorial exploratorio se consideró el método de extracción de componentes principales y rotación varimax. Se consideró eliminar aquellos ítems cuyas cargas factoriales fueran menores a 0,30. Luego, con los instrumentos ya depurados, se procedió a calcular el alfa de Cronbach de las puntuaciones totales y de cada sub-escala de ambos instrumentos, con sus respectivos intervalos de confianza (IC) al 95\%. Posteriormente, se calcularon los estadísticos descriptivos y la prueba de normalidad de Kolmogorov-Smirnov para cada variable y según sexo y grado escolar. Seguidamente, y considerando una distribución no normal de las puntuaciones de todas las variables en la muestra total y en todos los grupos sujetos a comparación, se empleó la prueba $U$ de Mann-Whitney para realizar la comparación según sexo y género. En las comparaciones en que se obtuvieron diferencias estadísticamente significativas se calculó también la probabilidad estimada de superioridad $\left(\mathrm{PS}_{\text {est }}\right)$ (Grissom, 1994) para estimar el tamaño del efecto mediante los criterios indicados por Grissom (1994). Finalmente, se calcularon las correlaciones Spearman entre las variables de personalidad y de adicción a las redes para la muestra total.

\section{RESULTADOS}

Resultados psicométricos.

El CPE obtuvo un alfa de .875. Las correlaciones corregidas ítem-puntuación total fueron mayores que 0,20, excepto en los ítems 13,19 y 21 (correlaciones de $0,156,0,189$ y $-0,074$, respectivamente), por lo que se decidió retirar dichos ítems del instrumento. El valor de la prueba KMO fue 0,894 y el de la prueba de Bartlett fue 2271,323 ( $\mathrm{gl}=190 ; \mathrm{p}<0,001)$, valores adecuados para proseguir con el análisis factorial exploratorio. Este análisis arrojó inicialmente cinco factores que explicaban el 60,45\% de la varianza. Esta estructura no resultó del todo convincente desde un punto de vista conceptual ya que creaba un factor en que se incluía ítems relacionados con el esfuerzo en los estudios y aspectos de autoestima; por lo que se decidió probar la estructura de cuatro ítems originalmente propuesta por los autores. En este nuevo análisis, se produjeron cuatro factores que explicaban el $55.40 \%$ de la varianza y en los cuales la distribución de los ítems fue prácticamente la misma que en la versión española original del instrumento, a excepción de los ítems 4 (que pasa de autoestima a autorrealización social) y 17 (que pasa de autoeficacia resolutiva a autoestima). Esta versión revisada tuvo un alfa de 0,900 (IC: 0,8820,915). Los alfas con sus respectivos IC de las escalas fueron los siguientes: autorrealización académica, AA (alfa=0,822; IC: 0,789-0,850); autoestima, AU (alfa=0,762; IC: 0,714-0,802); autorrealización social, AS (alfa $=0,784$; IC: $0,740-0,820)$ y autoeficacia resolutiva, AR (alfa $=0,726$, IC: 0,671-0,772).

El CARS obtuvo un alfa de 0,937 . Todos los ítems presentaron correlaciones corregidas con la puntuación total mayores que 0,20. El valor de la prueba KMO fue de 0,934 , en tanto que el de la prueba de Bartlett fue de 3707,092 ( $\mathrm{gl}=276 ; \mathrm{p}<0,001)$. Dados estos valores, se continuó con el análisis factorial exploratorio. Éste arrojó cuatro factores que explicaban el $57,52 \%$ de la varianza; sin embargo, este modelo fue desechado por que uno de los factores contenía un solo ítem. Se probó con una estructura de tres factores, a fin de tratar de replicar la estructura trifactorial original del instrumento. Los tres factores explicaron el 53,13 de la varianza, sin embargo, el número de ítems era muy desigual de un factor al otro. Se realizó una nueva prueba, con una estructura de cinco factores, los cuales explicaron el $61,54 \%$ de la varianza. Se consideró que esta estructura era más coherente conceptualmente que la de tres factores obtenida en la presente muestra. Los alfas de las puntuaciones de las escalas y sus

Tabla 2. Estadísticos descriptivos y prueba de Kolmogorov-Smirnov de normalidad de las variables en la muestra total

\begin{tabular}{lcccc}
\hline Variables & $\boldsymbol{M}$ & $\boldsymbol{D} \boldsymbol{E}$ & $\boldsymbol{K}-\boldsymbol{S}$ & Sig. \\
\hline AA & 23,7 & 5,49 & 0,063 & 0,005 \\
AU & 12,13 & 3,19 & 0,139 & $<0,001$ \\
AS & 10,57 & 3,47 & 0,103 & $<0,001$ \\
AR & 10,99 & 3,13 & 0,119 & $<0,001$ \\
NC & 11,90 & 8,22 & 0,106 & $<0,001$ \\
PR & 5,86 & 5,47 & 0,170 & $<0,001$ \\
FC & 3,94 & 3,03 & 0,117 & $<0,001$ \\
TE & 4,12 & 3,05 & 0,097 & $<0,001$ \\
OB & 5,03 & 3,17 & 0,090 & $<0,001$ \\
\hline
\end{tabular}

Nota: $M=$ Media; $D E=$ Desviación estándar; $K-S=$ Estadístico de la prueba de Kolmogorov-Smirnov; AA = Autorrealización académica; $\mathrm{AU}=$ Autoestima; $\mathrm{AS}=$ Autorrealización social; $\mathrm{AR}=$ Autoeficacia resolutiva; $\mathrm{NC}=$ Necesidad de conexión; $\mathrm{PR}=$ Predominancia $; \mathrm{FC}=$ Falta de control; $\mathrm{TE}=$ Tiempo excesivo $\mathrm{OB}=$ Obsesión por las redes sociales. 
Tabla 3. Estadísticos descriptivos y prueba de Kolmogorov-Smirnov de normalidad de las variables según sexo

\begin{tabular}{|c|c|c|c|c|c|c|c|c|}
\hline \multirow[b]{2}{*}{ Variables } & \multicolumn{4}{|c|}{ Hombres } & \multicolumn{4}{|c|}{ Mujeres } \\
\hline & $M$ & $D E$ & K-S & $p$ & $M$ & $D E$ & K-S & $p$ \\
\hline AA & 23,00 & 4,77 & 0,128 & 0,001 & 23,24 & 5,74 & 0,082 & 0,001 \\
\hline $\mathrm{AU}$ & 12,53 & 2,61 & 0,152 & $<0,001$ & 11,96 & 3,38 & 0,144 & $<0,001$ \\
\hline AS & 11,09 & 3,00 & 0,101 & 0,031 & 10,36 & 3,63 & 0,107 & $<0,001$ \\
\hline $\mathrm{AR}$ & 11,58 & 2,42 & 0,127 & 0,002 & 10,77 & 3,35 & 0,112 & $<0,001$ \\
\hline $\mathrm{NC}$ & 14,65 & 8,34 & 0,076 & 0,200 & 10,82 & 7,95 & 0,118 & $<0,001$ \\
\hline PR & 6,93 & 5,56 & 0,167 & $<0,001$ & 5,43 & 5,39 & 0,188 & $<0,001$ \\
\hline $\mathrm{FC}$ & 4,65 & 3,15 & 0,102 & 0,027 & 3,66 & 2,94 & 0,134 & $<0,001$ \\
\hline $\mathrm{TE}$ & 4,52 & 2,58 & 0,099 & 0,037 & 3,97 & 3.21 & 0,119 & $<0,001$ \\
\hline $\mathrm{OB}$ & 5,18 & 2,78 & 0,118 & 0,005 & 4,97 & 3.31 & 0,109 & $<0,001$ \\
\hline
\end{tabular}

Tabla 4. Prueba U de Mann-Whitney de comparación de las variables según sexo

\begin{tabular}{lcccccc}
\hline Variables & Sexo & Rango Promedio & $\boldsymbol{U}$ & $\boldsymbol{Z}$ & $\boldsymbol{p}$ & $\boldsymbol{P \boldsymbol { S } _ { \text { est } }}$ \\
\hline AA & Hombres & 149,65 & 9128,50 & $-0,356$ & 0,722 & - \\
& Mujeres & 153,63 & & & & \\
\multirow{2}{*}{ AU } & Hombres & 159,26 & 8793,00 & $-0,847$ & 0,397 & - \\
& Mujeres & 149,83 & & & & \\
AS & Hombres & 164,02 & 8383,00 & $-1,442$ & 0,149 & - \\
& Mujeres & 147,95 & & & & \\
AR & Hombres & 166,34 & 8184,00 & $-1,733$ & 0,083 & - \\
& Mujeres & 147,04 & & & & \\
NC & Hombres & 181,78 & 6855,50 & $-3,652$ & $<0,001$ & 0,36 \\
& Mujeres & 140,95 & & & & \\
PR & Hombres & 171,84 & 7710,50 & $-2,421$ & 0,015 & 0,41 \\
& Mujeres & 144,87 & & & & \\
FC & Hombres & 172,35 & 7666,50 & $-2,490$ & 0,013 & 0,41 \\
& Mujeres & 144.67 & & & & \\
TE & Hombres & 168,87 & 7966,50 & $-2,051$ & 0,040 & 0,42 \\
& Mujeres & 146,04 & & & & \\
OB & Hombres & 157,48 & 8946,00 & $-0,623$ & 0,534 & - \\
\hline
\end{tabular}

Nota: $U=\mathrm{U}$ de Mann-Whitney; $Z=$ Estadístico $Z ; P S$ = Probabilidad estimada de superioridad; AA = Autorrealización académica; $\mathrm{AU}=$ Autoestima; $\mathrm{AS}=$ Autorrealización social; $\mathrm{AR}=$ Autoeficacia resolutiva; $\mathrm{NC}=$ Necesidad de conexión; $\mathrm{PR}=$ Predominancia; $\mathrm{FC}=$ Falta de control; $\mathrm{TE}=$ Tiempo excesivo; $\mathrm{OB}=$ Obsesión por las redes sociales.

respectivos IC fueron los siguientes: necesidad de estar conectado, $\mathrm{NC}$ (alfa=0,892; IC: 0,872-0,909); predominancia, PR (alfa $=0,855$; IC: $0,827-0,878$ ); falta de control, FC (alfa=0,718; IC: 0,657-0,768); tiempo excesivo, TE (alfa $=0,684$; IC: $0,616-0,740)$ y obsesión por las redes sociales, $\mathrm{OB}(\mathrm{alfa}=0,658 ; \mathrm{IC}$ : $0,584-0,719)$.

\section{Descriptivos y comparacione.}

En la tabla 2 se presentan los datos descriptivos y los resultados de la prueba de Kolmogorov-Smirnov para todas las variables en la muestra total. Según esta prueba, todas las variables tienen distribución no normal. Asimismo, la tabla 3 presenta los descriptivos $\mathrm{y}$ resultados de la prueba de Kolmogorov-Smirnov 
Tabla 5. Correlaciones Spearman entre las variables de personalidad eficaz y adicción a las redes sociales

\begin{tabular}{lccccc}
\hline & & $\mathbf{A A}$ & $\mathbf{A U}$ & $\mathbf{A S}$ & $\mathbf{A R}$ \\
\hline \multirow{2}{*}{ NC } & Rho & $-0,211$ & $-0,182$ & $-0,145$ & $-0,142$ \\
& $p$ & $<0,001$ & 0,001 & 0,011 & 0,013 \\
PR & $R h o$ & $-0,311$ & $-0,236$ & $-0,206$ & $-0,193$ \\
& $p$ & $<0,001$ & $<0,001$ & $<0,001$ & 0,001 \\
FC & $R h o$ & $-0,111$ & $-0,133$ & $-0,084$ & $-0,088$ \\
TE & $p$ & 0,053 & 0,020 & 0,144 & 0,125 \\
& $R h o$ & $-0,247$ & $-0,216$ & $-0,208$ & $-0,117$ \\
OB & $p$ & $<0,001$ & $<0,001$ & $<0,001$ & 0,042 \\
& $R h o$ & $-0,030$ & $-0,056$ & -.011 & -.008 \\
& $p$ & 0,607 & 0,332 & .850 & .887 \\
\hline
\end{tabular}

Nota: $\mathrm{AA}=$ Autorrealización académica; $\mathrm{AU}=$ Autoestima; $\mathrm{AS}=$ Autorrealización social; $\mathrm{AR}=$ Autoeficacia resolutiva; $\mathrm{NC}=$ Necesidad de conexión $; \mathrm{PR}=$ Predominancia; $\mathrm{FC}=$ Falta de control; $\mathrm{TE}=$ Tiempo excesivo; $\mathrm{OB}=$ Obsesión por las redes sociales.

según sexo. Todas las variables tienen distribución no normal en todos los grupos, excepto la variable necesidad de conectarse en el grupo masculino. En la Tabla 4 se presentan los resultados de la prueba $U$ de Mann-Whitney de comparación según sexo. Como puede verse, no se aprecian diferencias significativas entre hombres y mujeres en las variables de personalidad eficaz, pero sí en casi todas las variables de adicción a las redes sociales. Los hombres puntúan significativamente más alto en necesidad de conectarse, predominancia, falta de control y tiempo excesivo. Sin embargo, aun en esos casos, el tamaño del efecto es nulo $\left(P S_{\text {est }}<0,50\right)$.

\section{Correlaciones}

En la tabla 5 se presentan las correlaciones Spearman entre las variables de personalidad eficaz y las de adicción a las redes sociales. Todas las correlaciones resultaron negativas, como se esperaba, pero no todas ellas fueron significativas. Destacan los siguientes hechos. El factor de obsesión con las redes sociales no muestra asociación con ninguna variable personalidad eficaz y el factor de falta de control está asociado sólo con autoestima. En cambio, necesidad de conexión, predominancia y tiempo excesivo están asociados con todas las variables de personalidad eficaz.

\section{DISCUSIÓN}

Respecto del CPE, es de notar que la estructura factorial obtenida en la presente muestra es bastante similar a la que obtuvieron Dapelo et al. (2006), con la sola excepción de la eliminación de tres ítems de la escala de asutorrealización social y el cambio de factor de dos ítems. De este modo, se confirma la estructura tetrafactorial originalmente propuesta por los creadores del CPE (Martín del Buey et al., 2004), aunque los factores hallados por Dapelo et al., (2006) en estudiantes chilenos y los obtenidos en el presente estudio no son exactamente los mismos que los del estudio de Martín del Buey et al., (2004). La porcentaje de varianza explicada por estos cuatro factores fue también muy similar al obtenido por Dapelo et al. La estructura tetrafactorial finalmente adoptada en el presente estudio se consideró conceptualmente más consistente que la de cinco factores, obtenida en un primer proceso de análisis factorial exploratorio. Las puntuaciones total y de las cuatro escalas obtenidas mostraron altos valores del alfa de Cronbach, igualmente similares a los obtenidos por Dapelo et al., y en todos los casos superiores a 0,60 ; lo que permite considerar a dichas puntuaciones como bastante confiables.

En cuanto al CARS, tal como se indicó, desechamos la estructura tetrafactorial obtenida por análisis factorial exploratorio por contener un factor con un solo ítem y la trifactorial porque muchos ítems se agrupaban en un solo factor, lo que, en nuestro juicio no permitía deslindar claramente dimensiones que resultaran útiles desde un punto de vista conceptual. Por consiguiente, se probó con una estructura de cinco factores que resultó mucho más satisfactoria. En otros estudios en escolares peruanos también se ha encontrado cierta dificultad de establecer una estructura multifactorial interpretable (e. g. Cortés, 2019). Los cinco factores obtenidos no sólo muestran una distribución más equitativa en cuanto al número 
de ítems, sino que parecen también más coherentes conceptualmente. El primer factor, necesidad de conectarse (NE) se refiere a la necesidad manifestada por el participante de establecer la conexión, permanecer conectado y a la vez la sensación de cada vez necesitar más tiempo de permanecer conectado. Alude también a estados psicológicos desagradables cuando se está conectado y al alivio que se siente al usar las redes sociales. El segundo factor, predominancia (PR), se refiere a la prioridad otorgada al uso de las redes sociales por encima de otras actividades en el ámbito familiar, social y académico. El tercer factor, falta de control (FC), tiene que ver con la sensación de descontrol en el tiempo dedicado a las redes sociales. El cuarto factor, tiempo excesivo (TE) se refiere a la conciencia por parte del participante, de la cantidad de tiempo que invierte en las redes sociales. El quinto factor, obsesión por las redes sociales (OB), se refiere a enfocar su atención y pensamientos a la actividad de participar en las redes sociales en los momentos en que no se está conectado. Aunque esta estructura parece conceptualmente más sólida, no resultó del todo satisfactoria desde el punto de vista de la confiabilidad de las puntuaciones. Sólo en el caso de las escalas $\mathrm{NC}$ y PR las puntuaciones exhiben altos niveles de confiabilidad, incluso considerando los intervalos de confianza, en tanto que las puntuaciones de la escala FC se acerca a este criterio. En el caso de las escalas TE y OB, especialmente ésta última, las puntuaciones no son totalmente satisfactorias, por lo que, en todo caso, la interpretación de cualquier resultado de las mismas debe realizarse con precaución.

Los análisis comparativos en función del sexo, indican que no hay diferencias entre hombres y mujeres en las variables de personalidad eficaz. Algunos estudios indican diferencias en algunas de las dimensiones del CPE; por ejemplo, en el estudio de Castellanos, Guerra y Bueno (2014), en universitarios chilenos, se halló que los hombres puntuaban significativamente más alto en autoestima y autoeficacia resolutiva, pero las mujeres ostentaban una puntuación más alta en autorrealización académica. En un estudio en adultos españoles (de 30 a 60 años) tampoco se encontraron diferencias entre sexos (Guerra, Arnaiz y Di Giusto, 2014). Al parecer, los factores que constituyen la personalidad eficaz se encuentran igualmente desarrollados en hombres y en mujeres, y este parece ser un rasgo contrastable a través de diversas culturas y edades.

En cambio sí se hallaron diferencias entre hombres y mujeres en la mayoría de las variables de adicción a las redes sociales. El factor en que no se encontró tales diferencias es el de obsesión por las redes sociales, tal vez debido a la confiabilidad relativamente baja de las puntuaciones de esa escala. En las demás variables la puntuación de los hombres fue significativamente más alta; sin embargo, el tamaño del efecto, considerando el criterio de Grissom (1994) fue nulo. Es decir, en la práctica, tales diferencias podrían ignorarse. En realidad, algunos estudios realizados en otras latitudes en personas jóvenes (e. g. Cam y Isbulan, 2012) muestran un mayor nivel de adicción a las redes sociales entre los hombres; sin embargo, esta no parece ser la norma. En su estudio en escolares peruanos, Cortés (2019) no halló diferencias entre sexos en la puntuación global del CARS. En algunos otros estudios tampoco se ha encontrado diferencias de sexo en distintos indicadores de adicción a las redes sociales, tanto en escolares como en universitarios (e.g., Araujo, 2016; Pontes, 2017) o bien se ha encontrado significancia estadística pero no práctica (VallejosFlores, Copez-Lonzoy y Capa-Luque, 2018). Floro y Siomos (2013) explican que los adolescentes de ambos sexos manifiestan mucho interés por las redes sociales y este hecho podría dar razón de que no se encuentren diferencias entre hombres y mujeres en estas variables. Sin embargo, tales resultados no son unánimes, ya que también existen muchos estudios que indican un mayor nivel de adicción a las redes sociales entre las mujeres (e. g., Andreassen, et al., 2016; Andreassen, Pallesen y Griffiths, 2017), o por lo menos un mayor nivel de urgencia por conectarse (Hormes, Kearns y Timko, 2014). En estos casos, una explicación podría ser que las mujeres deben soportar un mayor nivel de estrés que los hombres, haciéndolas más propensas que éstos a adquirir una adicción a las redes sociales (Kuss y Griffiths, 2011). Por otro lado, en este campo como en muchos otros en investigación psicosocial, los resultados respecto al comportamiento de determinadas variables pueden variar ampliamente de un contexto a otro, dependiendo incluso de los instrumentos e indicadores considerados en la medición.

Como se esperaba, se hallaron correlaciones negativas entre las variables de adicción a las redes sociales y las de personalidad eficaz. En general, se reconoce que factores como la autoestima y la autoeficacia guardan una estrecha relación con indicadores de bienestar psicológico y de este modo, una relación negativa con diversos tipos de desajuste. Existe evidencia de relaciones negativas entre autoestima y adicción a las redes sociales (Blachnio et al., 2016; Hawi y Samaha, 2017; 
Milosevic y Zezelj, 2014). Se ha sostenido que el uso adictivo de las redes sociales podría estar motivado por el deseo de inhibir sentimientos de baja autoestima (Andreassen et al., 2017), de ser así, es de esperar que los individuos de baja autoestima sean más propensos a adquirir esta adicción. Por otro lado, la baja autoestima también está relacionada con conductas de "autopromoción" mediante una mayor actividad on line (Mehdizadeh, 2010), lo que podría constituirse en un factor de riesgo para una actividad adictiva en las redes sociales. También existe cierta evidencia indirecta sobre el papel de la autoeficacia; por ejemplo, la adicción a internet está negativamente relacionada con la autoeficacia social y locus de control interno en universitarios (Iskender y Akin, 2010). Específicamente sobre la adicción a las redes sociales, Milosevic y Zezelj (2014) también hallaron que se hallaba relacionada con una baja autoeficacia. Este tipo de resultados ha sido previamente relacionado con la llamada "hipótesis de compensación" es decir, que "las personas menos adaptadas a las interacciones de la vida real buscan logros en el mundo online exhibiendo más tendencias adictivas" (Milosevic y Zezelj, 2014, p. 233). En general, parece que factores que, como la baja autoestima y la baja autoeficacia, tradicionalmente han sido relacionados con diversos tipos de disfunciones y emociones displacenteras, como la ansiedad, estrés crónico y depresión, guardan fuertes relaciones con otros tipos de disfunciones, como ciertos tipos de adicciones, en la medida que las adicciones mismas son también, en muchos casos, una vía de escape frente a dichas emociones displacenteras.

De los cinco factores de indicadores a las redes sociales, sólo aquellos cuyas puntuaciones mostraron mayor confiabilidad (necesidad de conexión y predominancia, están relacionados con las cuatro variables de personalidad eficaz, aunque también lo está el factor de tiempo excesivo, el cual mostró niveles casi en el límite de lo aceptable en la confiabilidad de sus puntuaciones. Por el contrario, otro factor, el de falta de control, con una confiabilidad relativamente alta, sólo correlacionó con autoestima. Estos resultados indicarían que la cuestión de la confiabilidad de las puntuaciones sólo en parte podría explicar el hecho de que estos factores muestren o no correlación la personalidad eficaz. Como ya señaló uno de los autores (Araujo, 2016), cada grupo de indicadores puede tener su propia dinámica de funcionamiento, lo que se vería reflejado no solamente en su manera particular de manifestarse en función de variables sociodemográficas, sino también en cuanto a cómo se relacionan con otras variables.
Consideramos que los presentes resultados son un primer paso hacia estudios con muestras de mayor tamaño y de una población más amplia, los cuales deberían permitir, en primer lugar, perfeccionar la información sobre las características psicométricas de los instrumentos aquí utilizados, en especial el CARS; y en segundo lugar, explorar la manera en que las relaciones aquí investigadas se ven moduladas por las variables sociodemográficas.

\section{Correspondencia}

Roberto Bueno Cuadra

Correo electrónico: rbueno@unfv.edu.pe

\section{REFERENCIAS BIBLIOGRAFICAS}

Andreassen, C. S. (2015). Online social network site addiction: A comprehensive review. Current Addiction Reports, 2, 175-184.

Andreassen, C. S., Billieux, J., Griffiths, M. D., Kuss, D. J., Demetrovics, Z., Mazzoni, E., \& Pallesen, S. (2016). The relationship between addictive use of social media and video games and symptoms of psychiatric disorders: A large-scale cross-sectional study. Psychology of Addictive Behaviors, 30(2), 252-262.

Andreassen, C.S., Tosheim, T., BrunBerg, G. S. \& Pallesen, S. (2012). Development of a Facebook addiction scale. Psychological Reports, 110, 501-517. Andreassen, C. S., Pallesen, S. \& Griffiths, M. D. (2017). The relationship between addictive use of social media, narcissism, and self-esteem: Findings from a large national survey. Addictive Behaviors, 64, 287-293.

Araujo, E. D. (2016). Indicadores de adicción a las redes sociales en universitarios de Lima. Revista Digital de Investigación en Docencia Universitaria, 10,48-58. doi: http://dx.doi.org/10.19083/ridu.10.494

Barros, S. M., Hernández, Y. C., Venegas, O. S., Cedillo, M. L., Alvarado, H. \& Cabrera, J.

A. (2017). Adolescentes de básica superior con adicción a internet y redes sociales y relaciones interpersonales. Revista Electrónica de Psicología Iztacala, 20, 42-68.

Bernal, C., (2010). Metodología de la investigación. Administración, economía, humanidades y ciencias sociales. Bogotá: Pearson Educación.

Błachnio, A., Przepiorka, A., \& Rudnicka, P. (2016). Narcissism and self-esteem as predictors of dimensions of Facebook use. Personality and Individual Differences, 90, 296-301.

Blachnio, A., Przepiorka, A., Senol-Durak, E. \& Durak, M. (2017). The role of personality

traits in Facebook and internet addictions: A study on Polish, Turkish, and Ukrainian samples. Computers in Human Behavior, 68, 269-275.

Boyd, D. M. \& Ellison, N. B. (2007). Social network 
sites: definition, history, and scholarship. Journal of Computer-Mediated Communication, 13, 210-230.

Bustamante, J. C., Salavera, C., Antoñanzas, L., Chueca, J., Teuel, P., Carrón, J., Larrosa, S. \& Bericat, C. (2014). La personalidad eficaz en el universitario y el uso del buen humor. International Journal of Developmental and Educational Psychology, 1, 467-476.

Cam, E., \& Isbulan, O. (2012). A new addiction for teacher candidates: Social networks. Turkish Online Journal of Educational Technology, 11, 14-19.

Caldevilla, D. (2010). Las redes sociales. Tipología, uso y consumo de las redes 2.0 en la sociedad digital actual. Documentación de las Ciencias de la Información, 33, 45-68.

Caplan, S. E. \& High, A. C. (2011). Online social interaction, psychosocial well-being, and problematic internet use. En: K. S. Young \& C. Nabuco de Abreu, (Eds.), Internet addiction. A handbook and guide to evaluation and treatment (pp. 35-53). Hoboken, NJ: Wiley.

Carbonell, X. (2015). ¿Qué son las adicciones tecnológicas? Internet, MMORPG y redes sociales. En: $\mathrm{X}$. Carbonell (Coord.) Adicciones tecnológicas: qué son y cómo tratarlas (pp. 13-36). Madrid: Síntesis.

Castellanos, S., Guerra, P., \& Bueno, J. A. (2014). Diferencias de género en personalidad eficaz en universitarios chilenos. International Journal of Developmental and Educational Psychology, 5, 131-140. Cayhan, A. A., \& Ceyhan, E. (2008). Loneliness, depression, and computer self-efficacy as predictors of problematic internet use. Cyberpsychology and Behavior, 11, 699-701.

Chóliz, M., \& Villanueva, V. (2011). Ellas, ellos y su móvil: uso, abuso (¿y dependencia?) del teléfono móvil en la adolescencia. Revista Española de Drogodependencias, $1,74-88$.

Cortés, F. (2019). Dificultades interpersonales y adicción a las redes sociales en adolescentes escolares de Lima. (Tesis de licenciatura. Universidad Peruana de Ciencias Aplicadas, Lima, Perú).

Dapelo, B., Marcone, R., Martín del Buey, F. de A., Martín, E. \& Fernández, A. (2006). Adaptación chilena del Cuestionario de Personalidad Eficaz para adolescentes. Psicothema, 18, 130-134.

Echeburúa, E. (2012). Factores de riesgo y de protección en la adicción a las nuevas tecnologías y redes sociales en jóvenes y adolescentes. Revista Española de Drogodependencias, 37, 435-447.

Echeburúa, E. \& de Corral, P. (2010). Adicción a las nuevas tecnologías y a las redes sociales en jóvenes: un nuevo reto. Adicciones, 22(2), 91-96.

Echeburúa, E. \& Requesens, A. (2012). Adicción a las redes sociales y nuevas tecnologías en niños $y$ adolescentes. Guía para educadores. Madrid: Pirámide. Eftekhar, A., Fullwood, C. \& Morris, N. (2014). Capturing personality from Facebook photos and photo- related activities: How much exposure do you need? Computers in Human Behavior, 37, 162-170.

Ehrenberg, A., Juckes, S., White, K. \& Walsh, S. P. (2008). Personality and self-esteem as predictors of young people's technology use. Cyberpsychology and Behavior, 11, 739-741.

Escurra, M. \& Salas, E. (2014). Construcción y validación del cuestionario de adicción a las redes sociales. Liberabit, 20, 73-91.

Fernández, N. (2013). Trastornos de conducta y redes sociales en internet. Salud Mental, 36, 521-527.

Floro, G. \& Siomos, K. (2013). The relationship between optimal parenting, Internet addiction and motives for social networking in adolescence. Psychiatry Research, 3, 529-534.

Franco, A. G., Martínez, E., Parra, C. A. \& Ramos, V. (2018). Identificación de rasgos de la personalidad que probabilizan la adicción a internet. PsicoEducativa: Reflexiones y Propuestas, 4, 30-35.

García, J. A. (2013). Adicciones tecnológicas: El auge de las redes sociales. Health and Addictions, 13, 5-14.

Gonzales, A. L., \& Hancock, J. T. (2011). Mirror, mirror on my Facebook wall: Effects of exposure to Facebook on self-esteem. CyberPsychology, Behavior, and Social Networking, 14, 79-83.

Griffiths, M. D., Kuss, D. J., \& Demetrovics, Z. (2014). Social networking addiction: An overview and preliminary findings. En: K. P. Rosenberg, \& . C. Feder (Eds.), Behavioral addictions. Criteria, evidence, and treatment (pp. 119-141). San Diego, CA: ElsevierAcademic Press.

Grissom, R. J. (1994). Probability of the superior outcome of one treatment over another, Journal of Applied Psychology, 79, 314-316.

Guerra, P., Arnaiz, A., \& Di Giusto, C. (2014). Las diferencias de género en personalidad eficaz en población adulta española. International Journal of Developmental and Educational Psychology, 5, 151-158.

Hawi, N. S. \& Samaha, M. (2017). The relations among social media addiction, self-esteem, and life satisfaction in university students. Social Science Computer Review, $35,576-586$.

Hong, F., Huang, D., Lin. H., \& Chiu, S. (2014). Analysis of the psychological traits, Facebook usage, and Facebook addiction model of Taiwanese university students. Telematics Inform,31, 597-606.

Hormes, J. M., Kearns, B. \& Timko, A. (2014). Craving Facebook? Behavioral addiction to online social networking and its association with emotion regulation deficits. Addiction, 109, 2079-2088.

Iskender, M. \& Akin, A. (2010). Social self-efficacy, academic locus of control, and internet addiction. Computers \& Education, 54, 1101-1106.

Kifafi, S., Lizana, V., \& Ortiz, R. (2012). Personalidad eficaz y rendimiento académico en estudiantes de séptimo y octavo año de educación básica. Revista de Psicología 
(Universidad de Viña del Mar), 2, 30-45.

Kuss, D. J. \& Griffiths, M. D. (2011). Addiction to social networks on the Internet: A literature review of empirical research. International Journal of Environment and Public Health, 8, 3528-3552.

Kuss, D. J. \& Griffiths, M. D. (2017). Social networking sites and addiction: Ten Lessons learned. International Journal of Environmental Research and Public Health, 14, 311. doi:10. 3390/ijerph14030311

Machin, D., Campbell, M. J., Tan, S. B. \& Tan, S. H. (2018). Sample sizes for clinical, laboratory and epidemiology studies. Hoboken, NJ: Wiley.

Martín del Buey, F., Martín, M. E., Dapelo, B., Pizarro, J. P., Di Gusto, C., Castellano, S. Arnaiz, A. (2015). El constructo personalidad eficaz: últimos avances. Revista de Orientación Educacional, 29, 55-70.

Martín del Buey, F., Martín, M. E. \& Fernández, A. (2009). El constructo "personalidad eficaz versus competente". International Journal of Developmental and Educational Psychology, 2, 575-583.

Martín del Buey, F., Martín, E, Fernández, A., Dapelo, B. \& Marcone, R. (2004). Evaluación de la personalidad eficaz en contextos educativos: primeros resultados. Revista de Orientación Educacional, 33-34, 79-101.

McCrae, R.R. \& Costa, P.T. (2006). Personality in adulthood. A five-factor theory perspective. Nueva York: The Guilford Press.

Mehdizadeh, S. (2010). Self-presentation 2.0: Narcissism and self-esteem on Facebook. Cyberpsychology, Behavior and Social Networking, 13, 357-364.

Milosevic, J. S. \& Zezelj, I. L. (2014). Psychological predictors of addictive social networking sites use: The case of Serbia. Computers in Human Behavior, 32, 229234.

Müller, K. W., Dreier, M., Beutel, M. E., Duven, E., Giralt, S., \& Wolfling, K. (2016). A hidden type of internet addiction? Intense and addictive use of social networking sites in adolescents. Computers in Human Behavior, 55, 172-177.

Niemz, K., Griffiths, M. \& Banyard, P. (2005). Prevalence of pathological internet use among university students and correlations with self-esteem, the General Health Questionnaire (GHQ), and disinhibition. Cyberpsychology and Behavior, 8, 562-570.
Pantic, I. (2014). Online social networking and mental health. Cyberpsychology, Behavior and Social Networking, 17, 652-657.

Pizarro, J. P., Martín, M. E., \& Cortés, V. (2012). Cuestionario de personalidad eficaz ampliado para niños y niñas de 8 a 12 años. Revista de Orientación Educacional, 26, 107-120.

Pontes, H. M. (2017). Investigating the differential effects of social networking site addiction and Internet gaming disorder on psychological health. Journal of Behavioral Addictions, 6, 601-610. doi: 10.1556/2006.6.2017.075 Ross, C., Orr, E. S., Sisic, M., Arsenault, J. M., Simmering, M. G. \& Orr, R. (2006).

Personality and motivations associated with Facebook use. Computers in Human Behavior, 25, 578-586.

Sanz, E., Alonso, R. A., Sáenz, M., Ponce de León, A. \& Valdemoros, M. A. (2018). Ocio, redes sociales y estudiantes españoles. Educación XXI, 21, 59-78.

Smith, E. R., Mackie, D. M. \& Claypool, H. M. (2014). Social psychology. New York: Psychology Press.

Steinfield, C., Ellison, N. B., \& Lampe, C. (2008). Social capital, self-esteem, and use of online social network sites: A longitudinal analysis. Journal of Applied Developmental Psychology, 29, 434-445.

Tamayo, M. (2003). El proceso de la investigación cientifica. Ciudad de México: Limusa.

Turel, O. \& Serenko, A. (2012) The benefits and dangers of enjoyment with social networking websites. European Journal of Inf Syst, 21, 512-528.

Vallejos-Flores, M. A., Copez-Lonzoy, A. \& CapaLuque, W. (2018). ¿Hay alguien en línea?: Validez y confiabilidad de la versión en español de la Bergen Facebook Addiction Scale (BFAS) en universitarios. Health and Addictions, 18, 175-184.

Wan, C. (2009). Gratifications \& loneliness as predictors of campus-SNS websites addiction \& usage pattern among Chines college students. Hong Kong: University of Hong Kong.

West, R. \& Brown, J. (2013). Theory of addiction (2da. Ed.). Chichester, Inglaterra: John Wiley \& Sons.

Wilson, K., Fornasier, S., \& White, K. M. (2010). Psychological predictors of young adults'use of social networking sites. Cyberpsychology, Behavior and Social Networking, 13, 173-177

Yesil, M.M. (2014). The relationship between facebook use and personality traits of University students. International Journal of Academic Research, 6(2), 7580 . 